\title{
Block of impulse propagation at an abrupt tissue expansion: evaluation of the critical strand diameter in 2- and 3-dimensional computer models
}

\author{
Vladimir G. Fast * , André G. Kléber \\ Department of Physiology, University of Berne, Bühlplaiz 5, CH-3012, Berne, Switzerland
}

Received 26 January 1995; accepted 12 May 1995

\begin{abstract}
Objective: Unidirectional conduction block in the heart can occur at a site where the impulse is transmitted from a small to a large tissue volume. The aim of this study was to evaluate the occurrence of conduction block in a 2-dimensional and 3-dimensional computer model of cardiac tissue consisting of a narrow strand abruptly emerging into a large area. In this structure, the strand diameter critical for the occurrence of block, $h_{c}$, was evaluated as a function of changes in the active and passive electrical properties of both the strand and the large medium. Methods: The effects of changes in the following parameters on $h_{c}$ were analysed: (1) maximum sodium conductance ( $\left.g N a_{\max }\right)$, (2) longitudinal $\left(R_{x}\right)$ and transverse $\left(R_{y}\right)$ intracellular resistivities, and (3) inhomogeneities in $g N a_{m u x}$ and $R_{x}$ and $R_{y}$ between the strand and the large area. Three ionic models for cardiac excitation described by Beelez-Reuter, Ebihara-Johnson, and Luo-Rudy ionic current kinetics were compared. Results: In the 2-dimensional simulations, $h_{c}$ was $175 \mu \mathrm{m}$ in Ebihara-Johnson and Beeler-Reuter models and $200 \mu \mathrm{m}$ in the Luo-Rudy model. At the critical strand diameter, the site of conduction block was located beyond the transition, i.e. a small circular area was activated in the large medium, whereas with narrower strands conduction block occurred within the strands. The decrease of $g N a_{\max }$ resulted in a large increase of $h_{c}$. This increase was mainly due to the change of $g N a_{m a x}$ in the large area, while $h_{c}$ was almost independent of $g N a_{\max }$ in the strand. Changing $R_{x}$ had no effect on $h_{c}$, whereas the increase of $R_{y}$ decreased $h_{c}$ and reversed conduction block. Inhomogeneous changes of $R_{x}$ and $R_{y}$ in the strand versus the large medium had opposite effects on $h_{c}$. When the resistivities of the strand alone were increased, $h_{c}$ also increased. In contrast, the increase of the resistivities in the large area reduced $h_{c}$. In the 3-dimensional model, $h_{c}$ was 2.7 times larger than the corresponding 2-dimensional values at the various levels of $\mathrm{gNa}_{\max }$ and resistivity. Conclusions: (1) At physiological values for active and passive electrical ptoperties, $h_{c}$ in the $2 \mathrm{D}$ simulations is close to $200 \mu \mathrm{m}$ in all three innic models. In the 3-dimensional simulations, $h_{c}$ is 2.7 larger than in the 2-dimensional models. (2) The excitable properties of the large area but not of the strand modify $h_{c}$. The decrease of intercellular coupling in the large medium facilitates impulse conduction and reduces $h_{c}$, while the same change in the strand increases $h_{r}$. (3) Occurrence of conduction block at an abrupt geometrical transition can be explained by both the impedance mismatch at the transition site and the critical curvature beyond the transition.
\end{abstract}

Keywords: Computer model; Conduction; Anisotropy

\section{Introduction}

Impulse conduction in cardiac tissue is dependent on the flow of local current at the front of the propagating wave. At a site of an abrupt geometrical expansion from a narrow cardiac strand into a large volume of myocardium, the amount of current supplied by the strand may not suffice to discharge the increased membrane surface downstream and conduction may fail (current-to-load mismatch). In the case where conduction is successful in the opposite direction (decrease of the electrical load across the transition) unidirectional conduction block (UDCB) will occur.
In the heart, a change in elcctrical load occurs in variety of locations including junctions between Purkinje fibers and ventricular muscle [1], between $S A$ node and atrial tissue [2], between accessory pathways and myocardium in the WPW syndrome [3], and between thin cell strands surviving within an infarcted myocardium and intact tissue [4]. The effect of a geometrical expansion on impulse conduction was studied theoretically in 1-dimensional cable models representing either an axon or a cardiac strand [5-10]. Experimentally, it was investigated in several tissue models including Purkinje-muscle junction [11-13], branching muscle bundles [14], an isthmus connecting two large areas of atrial or ventricular tissue $[3,15,16]$ and

\footnotetext{
* Corresponding author. Tel. (+41-31) 63187 07; Fax (+41-31) 631 4611 .
}

Time for primary review 27 days. 
patterned cell cultures with narrow strands abruptly diverging into a large cell area [17-19].

An important parameter in the geometry-dependent mechanism of UDCB is the critical diameter of a cardiac strand at which the block occurs. This parameter shares a close similarity with the critical size of excited tissue necessary to produce a propagated response (concept of liminal length [20-22]), with the critical width of an isthmus connecting two large excitable areas [16], and with the critical curvature of a propagating wave front [23,24]. The value of critical strand width has not been calculated in the computer simulations until now and its dependence on the passive and active model properties has not been established. Here we use a computer model of cardiac excitation to calculate the critical strand width and to evaluate its dependence on tissue properties. The specific goals were the following:

(1) To evaluate the critical strand size in 2-dimensional vs. 3-dimensional models. The electrical load imposed by the large tissue area on the narrow strand depends on the dimensionality of the large area. Thus, the critical strand width is expected to be different depending on whether the strand diverges into a thin 2-dimensional sheet of myocardium or into a 3-dimensional myocardial wall.

(2) To investigate the effects of inhomogeneities in tissue properties at the geometrical expansion on critical strand width. We modelled inhomogeneities in excitability and in resistivity between the strand and the large area. Such an inhomogeneity is present at the PM junction where both passive and excitable properties of the Purkinje fiber and of the ventricular muscle are different.

(3) To test whether the main findings about the critical strand width are model independent by using different models for the description of ionic currents in ventricular myocardium according to Beeler-Reuter [25], EbiharaJohnson [26], and Luo-Rudy [27].

\section{Methods}

The 2-dimensional (2D) model was designed to describe impulse propagation in a thin sheet of myocardium and was similar to the model used previously [19]. The extracellular medium was assumed to be isotropic and its resistance to be negligible. The model did not include the cellular structure, i.e. intercellular and cytoplasmic resistances were lumped together. However, the overall intracellular resistivity could be varied throughout the medium. The equation which describes propagation in the $2 \mathrm{D}$ model was the following:

$C_{m} \frac{\partial V}{\partial t}=\frac{1}{S_{v}}\left(\frac{\partial}{\partial x}\left(\frac{1}{R_{x}} \frac{\partial V}{\partial x}\right)+\frac{\partial}{\partial y}\left(\frac{1}{R_{y}} \frac{\partial V}{\partial y}\right)\right)-I_{i o n}$

where $V$ is membrane potential, $C_{m}$ is the specific membrane capacitance $\left(\mu \mathrm{F} / \mathrm{cm}^{2}\right), S_{v}$ is the surface-to-volume ratio, $I_{\text {ion }}$ is the total ionic current $\left(\mu \mathrm{A} / \mathrm{cm}^{2}\right), R_{x}$ and $R_{y}$ are internal resistivities in $x$ and $y$ directions $(\Omega \mathrm{cm})$.

In the $3 \mathrm{D}$ case, we considered a model with cylindrical symmetry. In this case, the $3 \mathrm{D}$ analog of eq. 1 can be

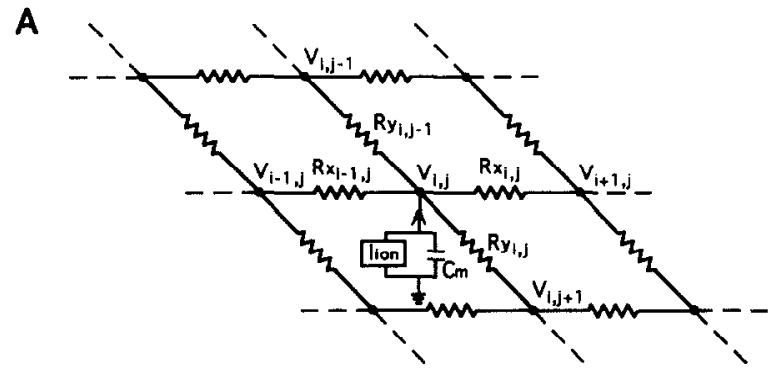

B

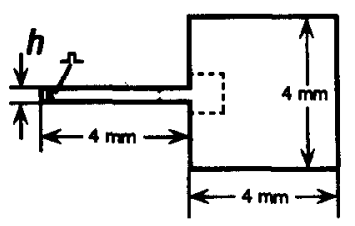

C

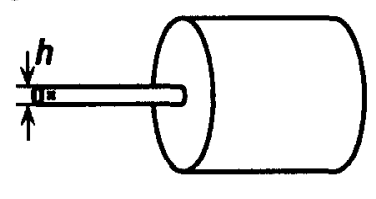

Fig. 1. (A) Electrical equivalent circuit of the anisotropic cardiac sheet. Membrane elements are represented as a capacitance $C_{m}$ in parallel with ionic current, $I_{i o n}$, described by either Ebihara-Johnson, Beeler-Reuter or Luo-Rudy ionic current models. $R_{x}$ and $R_{y}$ are resistive connections between excitable elements in $x$ and $y$ directions respectively. (B and $C$ ) 2-dimensional (B) and 3-dimensional (C) structures with an abrupt expansion of a narrow strand into a large medium. $h$ is a stand width (B) or a strand diameter (C). Asterisk indicates the site of stimulation. Only small portion of the medium (indicated by a dashed line in B) was used in subsequent Figures for the presentation of activation maps.

reduced to $2 \mathrm{D}$ equation and presented in the following form:

$C_{m} \frac{\partial V}{\partial t}=\frac{1}{S_{v}}\left(\frac{\partial}{\partial x}\left(\frac{1}{R_{x}} \frac{\partial V}{\partial x}\right)+\frac{1}{y} \frac{\partial}{\partial y}\left(y \frac{1}{R_{y}} \frac{\partial V}{\partial y}\right)\right)-I_{i o n}$

Here, $x$ corresponds to the axes of symmetry, and $y$ corresponds to the radial coordinate (see Fig. 1C).

To solve eq. 1 numerically, it was divided into isopotential excitable patches of the dimensions $\Delta x$ and $\Delta y$ (Fig. 1A). Substituting the right part of the eq. 1 with the difference approximation, the equation for membrane potential of the $2 \mathrm{D}$ patch with coordinates $i, j$ is the following:

$$
\begin{aligned}
C_{m} \frac{\partial V_{i, j}}{\partial t}= & \frac{1}{S_{v}}\left[K_{x ; i-1, j}\left(V_{i-1, j}-V_{i, j}\right)\right. \\
& -K_{x ; i, j}\left(V_{i, j}-V_{i+1, j}\right) \\
& +K_{y ; i, j-1}\left(V_{i, j}-V_{i, j-1}\right) \\
& \left.-K_{y ; i, j}\left(V_{i, j}-V_{i, j+1}\right)\right]-I_{i o n}
\end{aligned}
$$

where

$K_{x ; i, j}=\frac{1}{\Delta x^{2} R_{x ; i, j}}, \quad$ and $\quad K_{y ; i, j}=\frac{1}{\Delta y^{2} R_{y ; i, j}}$

describe the resistive links in $x$ and $y$ directions of the element $(i, j)$. The geometrical shape of the model and the pattern of resistive connections were coded by coefficients $K_{x ; i j}, K_{y ; i, j}$ which were calculated in advance and stored in arrays. The impermeable boundaries were modeled by setting $K_{x ; i, j}=0$ and $K_{y ; i, j}=0$ at the border elements. 
In the 3D case, the corresponding difference approximation is the following:

$$
\begin{aligned}
C_{m} \frac{\partial V_{i, j}}{\partial t}= & \frac{1}{S}\left[K_{\lambda, i \cdots 1, j}\left(V_{i \cdots 1, j}-V_{i, j}\right)\right. \\
& K_{x i, j, j}\left(V_{i, j} V_{i+1, j}\right) \\
& +\frac{1}{2 j-1}\left(K_{y: i, j \cdots 1}\left(V_{i, j}-V_{i, j \cdots 1}\right)\right. \\
& \left.\left.-K_{i, i, j}\left(V_{i, j}-V_{i, j+1}\right)\right)\right]-I_{i o n}
\end{aligned}
$$

where

$$
K_{x ; i, j}=\frac{1}{\Delta x^{2} R_{x i, j}}, \quad \text { and } \quad K_{y ; i, j}=\frac{j}{\Delta y^{2} R_{y ; i, j}} .
$$

The equations 3 and 4 were solved using the Peaceman-Rachford alternating-direction implicit algorithm [28]. Space integration steps $\Delta x, \Delta y$ were varied in the range from 10 to $50 \mu \mathrm{m}$ to keep the ratio between the electrotonic space constant and the space integration step at about 20. The ordinary differential equations for the ionic variables were solved by the Rush-Larsen algorithm [29,30]. The time integration step was in the range from 1 to $3 \mu \mathrm{s}$ depending on the value of maximum sodium conductance. Computer-generated lookup tables with $\Delta V=0.1 \mathrm{mV}$ were used to calculate rate constants.

The simulated structures are shown on Fig. $1 \mathrm{~B}$ and $\mathrm{C}$. The 2D structure consisted of a narrow strand of a width $h$ abruptly emerging into a large area. The corresponding 3D structure was obtained by rotation of the 2-dimensional structure around the axis of symmetry. In this case, $h$ corresponds to the diameter of the strand. The dimensions of the large area and the length of the strand were $4 \mathrm{~mm}$ which was about six times larger than electrotonic space constant. Simulations showed that the dimensions of the large area did not affect impulse propagation at the transition point when the dimensions were larger than two space constants. Impulse propagation was initiated at the left end of the strand by injecting current with double threshold strength. To determine the critical width, $h_{c}$, propagation was simulated in structures with gradually decreasing strand width and the value for last successful propagation was defined as $h_{c}$.

The value for $C_{m}=1.0 \mu \mathrm{F} / \mathrm{cm}^{2}$ and $S_{v}=0.25 \mu \mathrm{m}^{-1}$ were normally used in calculations. Three models describing the kinetics of ionic current $I_{\text {ion }}$ were used: the Beeler-Reuter model [25] referred to in the text as the BR model, the Ebihara-Johnson (EJ) model [26], and the Luo-Rudy (LR) model [27]. The reversal potential for the slow inward current was fixed at $+70 \mathrm{mV}$ in all models. The critical strand diameter was calculated as a function of maximal sodium conductance, $g N a_{\max }$, and the tissue resistivities, $R_{x}$ and $R_{y}$. From Eq. 1 follows that isotropic variation of tissue resistivities is equivalent to the change in surface-to-volume ratio $S_{v}$.

Activation times were determined at the moment when membrane potential reached the value of $-60 \mathrm{mV}$ which is close to the threshold potential for the sodium inward current in all models. Selection of this activation criterion was validated in our previous work [19]. These activation times were used for constructing isochrone activation maps. Simulations were programmed in FORTRAN and carried out on VAX-6410 with Vector Processor

\section{Results}

\subsection{Slow conduction and conduction block at the abrupt expansion}

Fig. 2 demonstrates impulse conduction in the 2D model with an abrupt expansion described by Luo-Rudy ionic current kinetics. In this simulation, the width of the strand $(200 \mu \mathrm{m})$ was slightly larger than the width necessary for conduction block to occur. Panel A shows an isochronal map of wave propagation across the transition region, Panel B shows the profile of conduction velocity, and Panel $\mathrm{C}$ shows the changes in action potential waveshapes. Far from the expansion in the strand, the excitation front was flat (A) and the impulse propagated with a uniform conduction velocity of $52.1 \mathrm{~cm} / \mathrm{s}$. Immediately before the transition, conduction slowed (B) and the excitation front became slightly curved. The most prominent changes in activation spread occurred immediately after the transition.
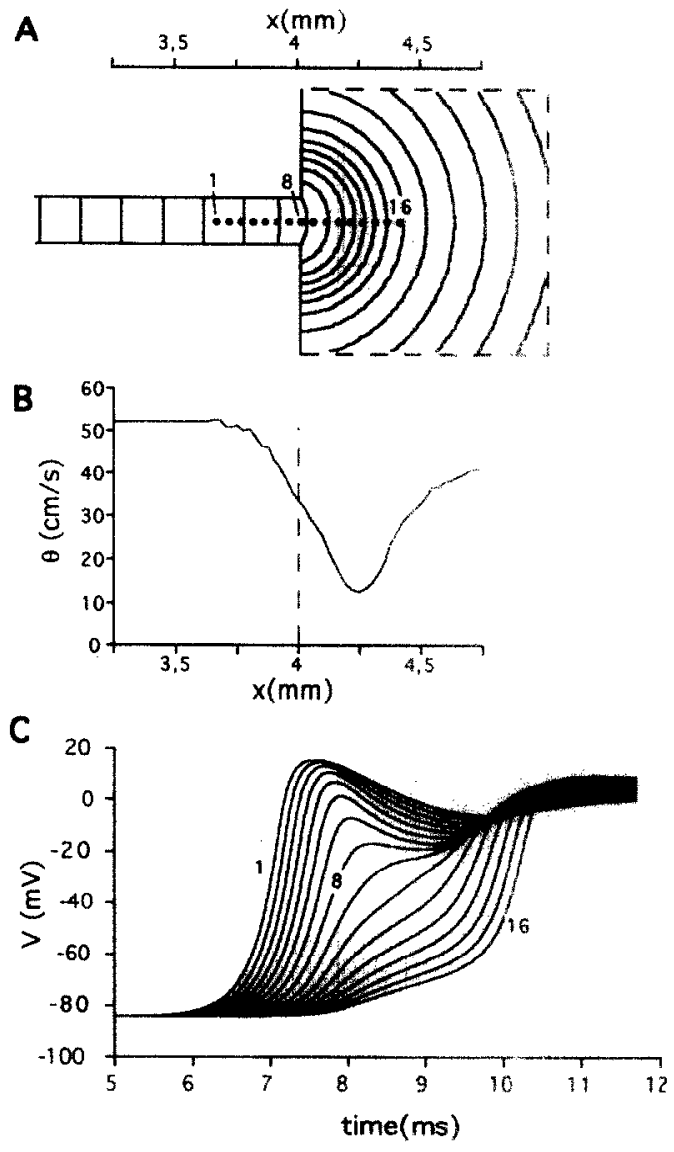

Fig. 2. Slowing of conduction at an abrupt expansion. (A) Isochrone map (interval $0.3 \mathrm{~ms}$ ) of wave propagation in a portion of medium indicated by the dashed line in $B$. Strand width $=200 \mu \mathrm{m}$. The distance, $x$, is measured from the left end of the strand. (B) Conduction velocity $(\theta)$ profile across the transition. (C) Recordings of action potentials $(V)$ at selected points depicted in A. Luo-Rudy model with $g N a_{\text {max }}=23$ $\mathrm{mS} / \mathrm{cm}^{2}, R_{r}=R_{n}=450 \Omega \mathrm{cm}$. 
The excitation front acquired a nearly-circular shape and conduction velocity decreased to $12.5 \mathrm{~cm} / \mathrm{s}$. The minimal conduction velocity was reached in the large area at a distance of $250 \mu \mathrm{m}$ from the transition. Afterwards, the conduction accelerated and slowly approached the value for conduction velocity in the strand. Action potential upstrokes $(\mathrm{C})$ had specific biphasic shapes with two rising phases typical for the geometrical expansion. Similar waveshapes were experimentally observed at PM junctions $[11,31,32]$ and at the junction between cultured cell strands and a large cell area [19].

The decrease of the strand width, $h$, to $175 \mu \mathrm{m}$ produced conduction block as shown on Fig. 3 while propagation was successful in the reversed direction (not shown). Voltage traces (Panel B) demonstrated that the action potential amplitude and duration gradually diminished across the transition. Such a gradual decrease of amplitude has been observed experimentally at a transition from a narrow cell strand into a large area in cultured cell monolayers using high-resolution optical recordings $[18,19]$. Because of the gradual changes in the voltage waveshapes, it was not possible to distinguish between actively generated responses and purely electrotonic potentials and therefore to locate conduction block. However, the site of conduction block could be determined from the traces of sodium inward current, iNa, depicted on Panel C. In contrast to the voltage changes, the amplitude of the sodium current first
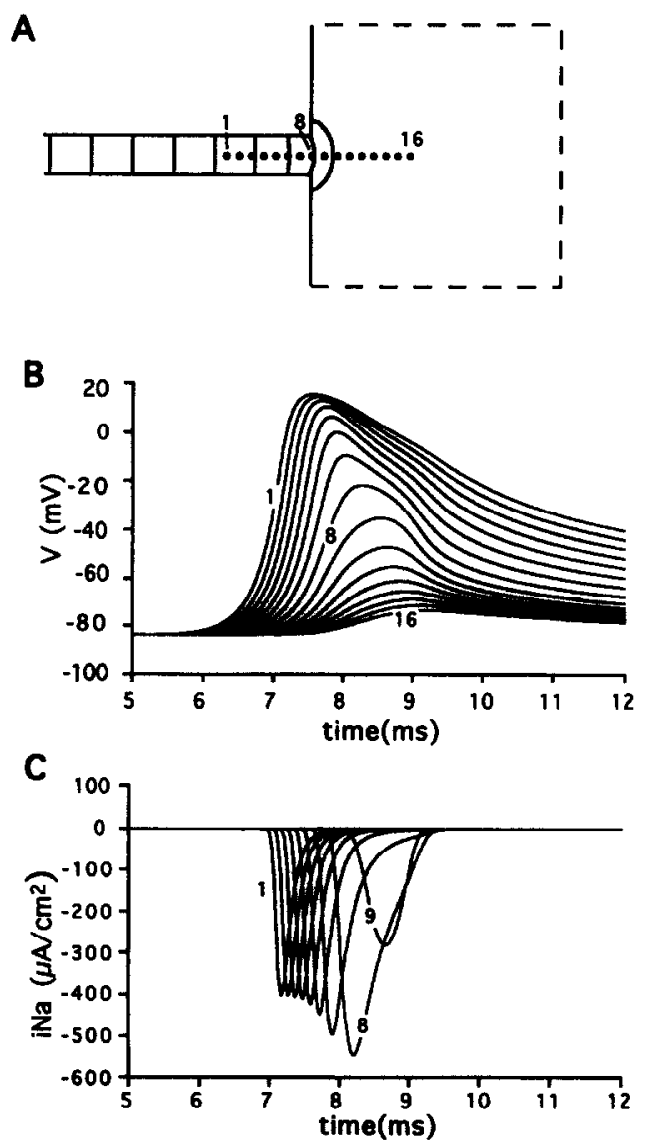

Fig. 3. Conduction block at abrupt expansion. Strand width $=175 \mu \mathrm{m}$. Other parameters are the same as on Fig. 2. (A) Isochrone map with interval of $0.3 \mathrm{~ms}$. (B and C) Recordings of action potentials $(V)$ and sodium current $(\mathrm{iNa})$ at the selected points depicted in $\mathrm{A}$.
A

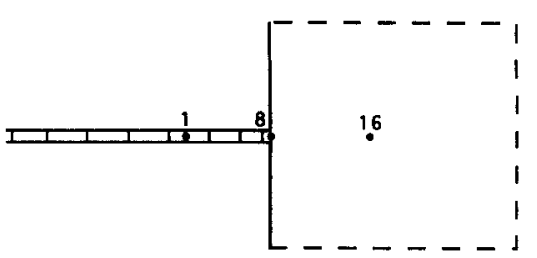

B

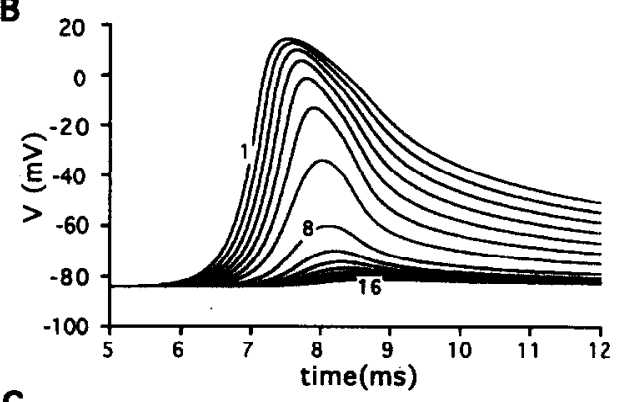

C

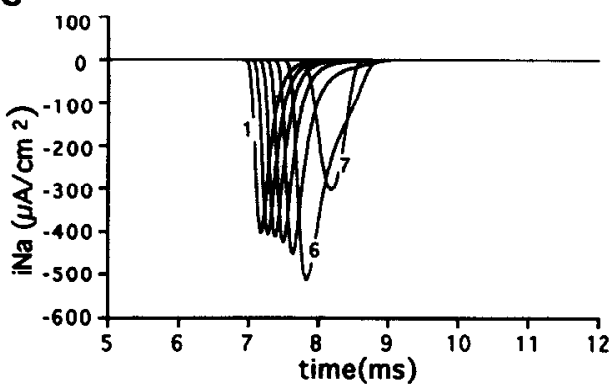

Fig. 4. Conduction block at a strand with the width of $50 \mu \mathrm{m}$. Other parameters are the same as on Fig. 2. (A) Isochrone map with interval of $0.3 \mathrm{~ms}$. (B and C) Recordings of action potentials $(V)$ and sodium current (iNa) at the selected points.

increased at the transition and then almost abruptly dropped to zero; no sodium current was activated at point 10 and beyond. The initial increase of the sodium current was caused by the slowing of the depolarisation rate near the transition which allowed more time for the activation of sodium current. As judged by the abrupt decrease of the current amplitude, conduction block occurred between points 9 and 10 at a distance between $50 \mu \mathrm{m}$ and $100 \mu \mathrm{m}$ from the expansion. The maximal membrane potential at these two points was $-35 \mathrm{mV}$ and $-47 \mathrm{mV}$ respectively. These values are slightly higher than the threshold potential in the LR model $(-60 \mathrm{mV})$. No sodium current was activated at sites 10 through 16 . Therefore, the voltage traces at these points were interpreted as purely electrotonic potentials.

Block of conduction from a strand of subcritical width ( $h=175 \mu \mathrm{m}$, Fig. 3) occurred beyond the transition, i.e. a small circular nucleus of excitation was present in the large area. As depicted on Fig. 4, the further decrease of the strand width to $50 \mu \mathrm{m}$ resulted in more proximal localisation of conduction block. In this case, more profound changes in action potential waveshapes and a more rapid decrement of conduction as compared to Fig. 3 were observed. The traces of the sodium current (Panel C) showed that the site of the conduction block was located between points 7 and 8 at a distance of $50 \mu \mathrm{m}$ before the transition. Therefore the activation wave did not leave the strand. 


\subsection{Conduction across an isthmus}

Cabo et al. recently investigated impulse conduction in a different structure consisting of a short isthmus connecting two large areas of ventricular myocardium [16]. To compare the results obtained at the geometrical expansion with the isthmus-type geometry we modelled the isthmus structure as illustrated on Fig. 5A. The length of the isthmus was fixed $(50 \mu \mathrm{m})$ and conduction was studied as the function of the isthmus width. Otherwise the model parameters were the same as for the simulations presented on Fig. 2-4 (Luo-Rudy ionic model). Several differences in impulse conduction across the isthmus were found with respect to the strand structure. Firstly, impulse conduction was blocked at a smaller isthmus width than in the case of the strand. The critical isthmus width was $150 \mu \mathrm{m}$ which is $25 \%$ smaller than the critical strand width. Secondly, the characteristics of conduction near the transition region were different. Fig. 5 shows impulse conduction across an isthmus with the critical width of $150 \mu \mathrm{m}$. Excitation was initiated by stimulating all elements at the left border of the model simultaneously. As in the strand, the excitation front was flat far from the transition (Panel $\mathrm{A}$ ) and parameters of propagation were the same as in the strand. In contrast to the strand, the activation front became concave before the isthmus (Panel $\mathrm{A}$ ), conduction accelerated (Panel B) and the action potential amplitude transiently increased
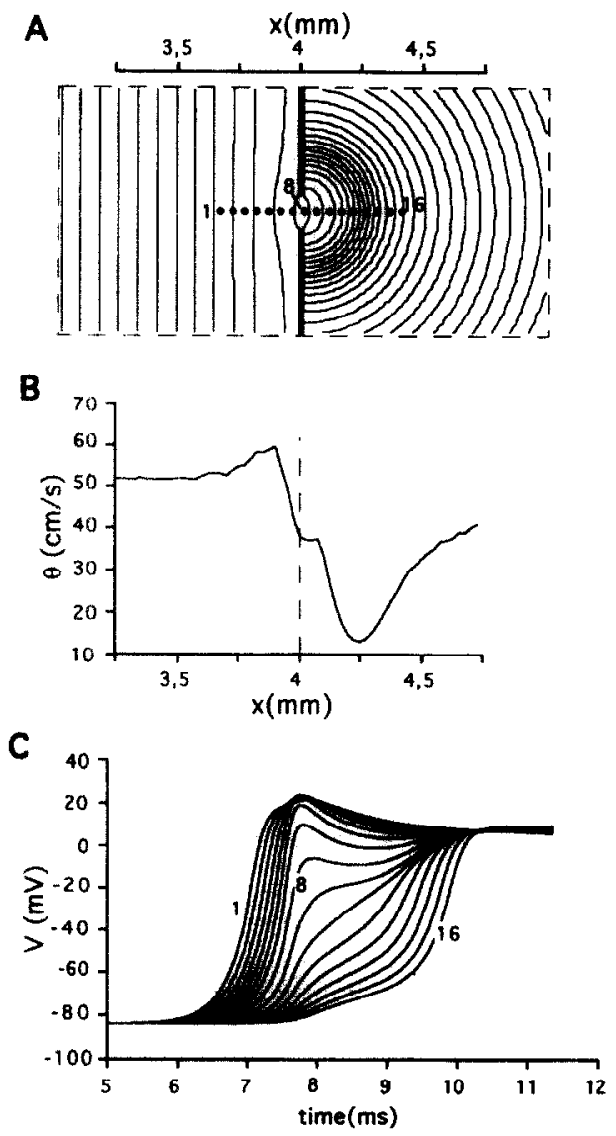

Fig. S. Impulsc conduction across an isthmus. Isthmus length $=50 \mu \mathrm{m}$, isthmus width $=150 \mu \mathrm{m}$. (A) Isochrone map of activation, isochrone interval of $0.15 \mathrm{~ms}$. The distance, $x$, is measured from the left end of the strand. (B) Conduction velocity ( $\theta$ ) profile across the transition. (C) Recordings of action potentials $(V)$ at the selected points depicted in $A$. Luo-Rudy model wilh $g N a_{\max }=23 \mathrm{mS} / \mathrm{cm}^{2}, R_{x}=R_{y}=450 \Omega \mathrm{cm}$.
Table 1

Critical strand width, $h_{c}$, in different ionic models

\begin{tabular}{lllll} 
Ionic model & $\begin{array}{l}g N a_{\max } \\
\left(\mathrm{mS} / \mathrm{cm}^{2}\right)\end{array}$ & $\begin{array}{l}d V / d t_{\max } \\
(\mathrm{V} / \mathrm{s})\end{array}$ & $\begin{array}{l}\theta_{6} \\
(\mathrm{~cm} / \mathrm{s})\end{array}$ & $\begin{array}{l}h \\
(\mu \mathrm{m})\end{array}$ \\
\hline EJ & 40 & 187.9 & 51.0 & 175 \\
LR & 23 & 189.5 & 52.1 & 200 \\
BR & 6.9 & 188.3 & 60)$. & 175 \\
\hline
\end{tabular}

EJ, LR, and BR indicate Ebihara-Johnson, Luo-Rudy, and Beeler-Reuter ionic models respectively; $g N a_{m a x}$ indicates maximum sodium conductance; $d V / d t_{\max }$ and $\theta_{o}$ indicate maximal upstroke rate of rise and conduction velocity measured during uniform propagation of a planar wave.

(Panel C). In the strand, conduction slowed and the amplitude decreased at the corresponding region (Fig. 2).

\subsection{Critical strand width in different ionic models}

Three ionic models are preferentially used in literature for the description of ionic currents in ventricular myocardium referred as Beeler-Reuter [25], Ebihara-Johnson [26], and Luo-Rudy [27] models. Therefore we compared transitional action potential waveshapes and values for critical strand width, $h_{c}$, in all three models and found similar results. Action potential upstrokes exhibited characteristic biphasic shapes during impulse conduction across geometrical expansion as shown on Fig. 2 in all three models. In each case, conduction block occurred at some value of the strand diameter which depended on the level of excitability. To compare the values for $h_{c}$ among the different models, the excitability of the models was adjusted to give comparable values of the maximal upstroke rate of rise, $d V / d V_{\max }$, during steady-state propagation. This meant increasing $\mathrm{gNa} a_{\max }$ from 23 to $40 \mathrm{mS} / \mathrm{cm}^{2}$ in the EJ model and from 4 to $6.9 \mathrm{mS} / \mathrm{cm}^{2}$ in the BR model to match $d V / d t_{\text {max }}$ of the Luo-Rudy model. The models were assumed to be isotropic and passive model parameters were set equal $(R=450 \Omega \mathrm{cm})$. Table 1 contains the results of calculations of $h_{c}$ in the three models along with $d V / d t_{\max }$ and conduction velocity, $\theta_{a}$ of a uniformly propagating planar wave. The accuracy of calculation of $h_{c}$ was determined by the size of the excitable elements which was $25 \mu \mathrm{m}$ in these simulations. It can be seen that the differences in $h_{c}$ between the three models were within the computational precision. Also, conduction velocity was essentially the same in EJ and LR models. The close results obtained in EJ and LR models are due to the similarity in the description of the fast sodium current in these two models (see Discussion). The conduction velocity in the BR model was about $20 \%$ larger than in the EJ and LR models at the comparable level of $d V / d t_{\text {mux }}$.

\subsection{Critical strand width in 3-dimensional model us. 2-dimensional model}

The electrical load imposed by the large tissue area on the narrow strand depends on the dimensionality of the large area. In 3-dimensional tissue the electrical load is larger than in the 2-dimensional tissuc. Thus $h_{c}$ is $\mathrm{cx}$ pected to be different depending on whether the strand diverges into a thin sheet of myocardium or into the 

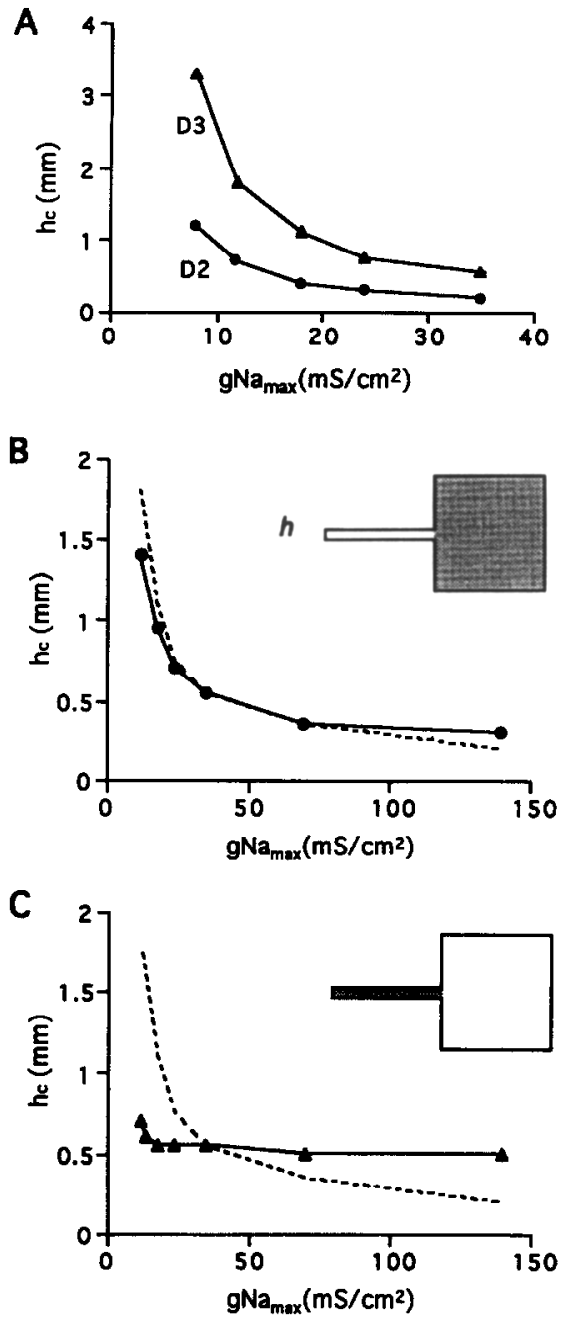

Fig. 6. Dependence of critical strand width, $h_{c}$, on maximum sodium conductance $g N a_{\max }$. (A) Effect of uniform change in $g N a_{\max }$ in the two- (D2) or three- (D3) dimensional models (EJ model with $R=450$ $\Omega \mathrm{cm}$ ). (B and C) Effect of a nonuniform change of $g N a_{\max }$ in the 2D model. Dashed curves correspond to the control when excitability was changed uniformly throughout the model. Shaded areas in the inset indicate the parts of the model where $\mathrm{gNa}_{\max }$ was altered: the large area (B) or the strand (C).

myocardial wall. In order to compare the $2 \mathrm{D}$ with $3 \mathrm{D}$ models, $h_{c}$ was calculated in 2D and 3D models at different values of excitability.

Fig. 6A shows the dependence of $h_{c}$ on maximum sodium conductance $g N a_{\max }$ for homogeneous $2 \mathrm{D}$ and $3 \mathrm{D}$ models described by $\mathrm{EJ}$ ionic current kinetics. For a $g N a_{\text {max }}=24 \mathrm{mS} / \mathrm{cm}^{2}$ used in the original formulation of the model [26], $h_{c}$ was 0.3 and $0.8 \mathrm{~mm}$ for $2 \mathrm{D}$ and $3 \mathrm{D}$ models, respectively. At $g N a_{\max }=35 \mathrm{mS} / \mathrm{cm}^{2}$ which more closely represents adult myocardium, $h_{c}$ was $0.2 \mathrm{~mm}$ (2D model) and $0.55 \mathrm{~mm}$ (3D model). A decrease of excitability to $8 \mathrm{mS} / \mathrm{cm}^{2}$ produced a rapid increase of $h_{c}$. The maximal values of $h_{c}$ reached in these simulations were $1.2 \mathrm{~mm}$ in the $2 \mathrm{D}$ case and $3.3 \mathrm{~mm}$ in the $3 \mathrm{D}$ case. At all values of $g N a_{\text {max }} h_{c}$ in the $3 \mathrm{D}$ model was larger than in the 2D model by a factor of 2.7 .

\subsection{Effect of inhomogeneity in $\mathrm{gNa}_{\max }$}

The success of impulse conduction across a tissue expansion depends on both the properties of the strand (source of current) and the large area (current sink). In order to estimate the relative contributions of the excitabilities of the strand and of the large area, we calculated $h_{c}$ upon changing $g N a_{\text {max }}$ either in the strand or in the large area. Panel B on Fig. 6 shows the effect of $g N a_{\max }$ changes in the large area alone in the $2 \mathrm{D}$ model described by EJ kinetics. In this case, the $g N a_{\max }$ in the strand was kept constant at a level of $35 \mathrm{mS} / \mathrm{cm}^{2}$. Panel $\mathrm{C}$ shows the effect of changes of $g N a_{\text {max }}$ in the strand when the excitability of the large area was constant. For comparison, the dashed lines represent the effect of a uniform change of $g N a_{\max }$ in the whole model. As shown on Panel B, $h_{c}$ was critically dependent on $\mathrm{gNa}_{\max }$ of the large area. By contrast, $h_{c}$ was almost independent of the excitability of the strand (C). This suggests that conduction block at the site of abrupt change of electrical load is mainly dependent on excitable properties of the cells distal to transition (large medium) and less on the excitability of the proximal cells (strand).

\subsection{Dependence of $h_{c}$ on axial resistivity}

In our previous work we have demonstrated that the increase of intracellular resistivity in the large area facilitated impulse conduction across a simulated geometrical expansion [19]. Here we further investigate this effect and evaluate the dependence of $h_{c}$ on intracellular resistivity and anisotropy in 2D and 3D models.

Dependence on resistivity in $2 D$ vs. $3 D$ models. The uniform increase of axial resistivity, $R$, facilitated impulse
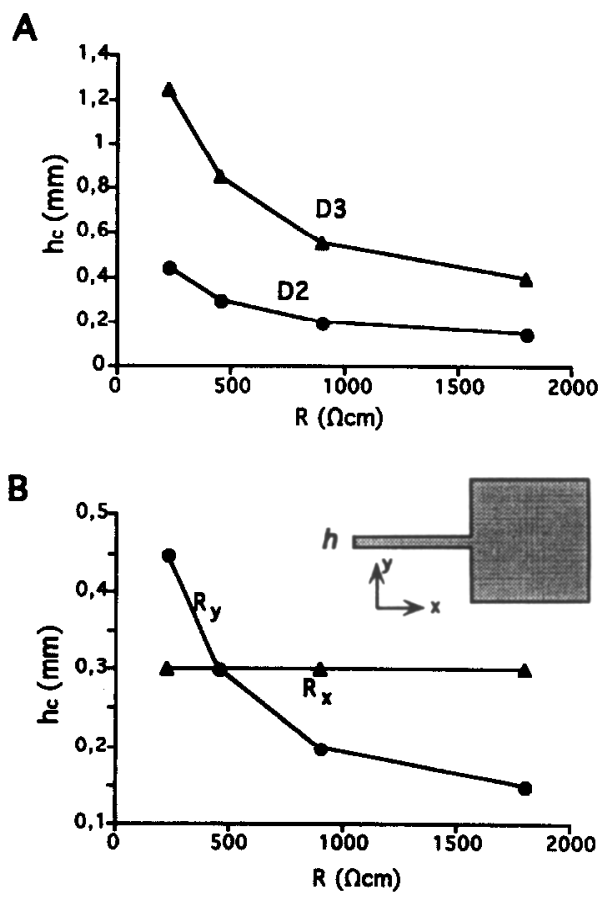

Fig. 7. Dependence of $h_{c}$ on intracellular resistivity $R$. (A) Effect of a uniform change in $R$ in isotropic 2D and 3D models (EJ model with $g N a_{\text {max }}=24 \mathrm{mS} / \mathrm{cm}^{2}$ ). (B) Effect of anisotropic change in $R$ in a uniform 2D model. The longitudinal resistivity, $R_{x}$, was changed in the whole model while transverse resistivity, $R_{y}$, was kept constant (curve " $R_{x}$ ") or, alternatively, $R_{y}$ was altered with constant $R_{x}$ (curve " $R_{y}$ "). The increase of transverse resistivity produced a decrease in $h_{c}$ while a change in longitudinal resistivity did not affect $h_{c}$. 

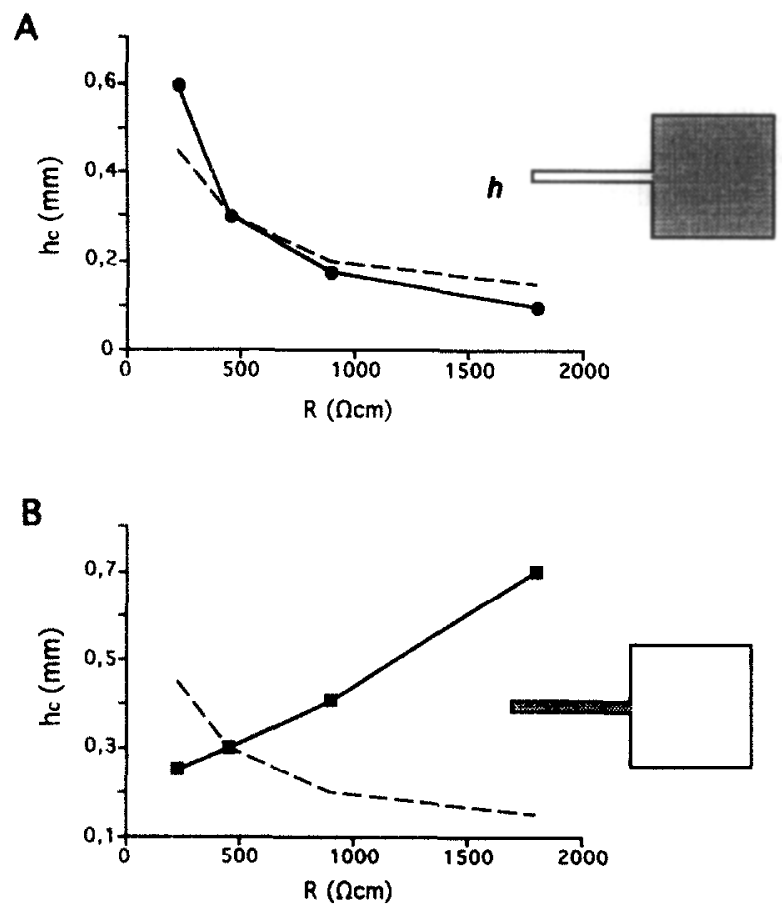

Fig. 8. Effect of a nonuniform change in intracellular resistivity on $h_{c}$. (A) Resistivity $R$ was varied in the large area. (B) Resistivity of the strand was changed. For comparison, the dashed curves correspond to the control when resistivity was changed uniformly throughout the model. Shaded areas in the insets indicate the parts of the model where $R$ was altered. EJ model with $g N a_{\max }=24 \mathrm{mS} / \mathrm{cm}^{2}$.

conduction across the expansion. When the strand width was narrow enough to produce conduction block at normal $R$, the increase of $R$ prevented the block. Conduction block was produced again at a smaller value of the strand width. Fig. 7A shows the dependence of $h_{c}$ on uniformly distributed resistivity in isotropic $2 \mathrm{D}$ and $3 \mathrm{D}$ models described by EJ kinetics with $g N a_{\max }=24 \mathrm{mS} / \mathrm{cm}^{2}$. Integration steps in these simulations were decreased with increasing intracellular resistivity, $R$, in order to keep a constant ratio $(\sim 20)$ between the electronic space constant and the space integration step (see Methods). The dependencies were well fitted by the inverse square-root relation $h_{c} \sim 1 / R^{1 / 2}$ in both $2 \mathrm{D}$ and $3 \mathrm{D}$ models. In the 3D model, $h_{c}$ was larger than that in the $2 \mathrm{D}$ model by a constant factor of 2.7. This factor was the same as in the case when $g N a_{\max }$ was varied (Fig. 6A).

Effect of anisotropy. Fig. 7B demonstrates the effect of an anisotropic change in the axial resistivity in both the strand and the large area. In the first case, the longitudinal resistivity $\left(R_{x}\right)$ was uniformly changed while transverse resistivity was kept constant $\left(R_{y}=450 \Omega \mathrm{cm}\right)$. On the second plot, the resistivity in the transverse direction $\left(R_{y}\right)$ was varied with constant $R_{x}$. Only the transverse resistivity affected $h_{c}$ while the longitudinal resistivity had no effect.

Inhomogeneous change of resistivity. Fig. 8 shows the effects of inhomogeneous changes in resistivity on $h_{c}$ in the isotropic 2D model. As depicted on Panel A, $h_{c}$ decreased when the resistivity of the large area alone was increased and the resistivity of the strand was kept con- stant $(450 \Omega \mathrm{cm})$. The dependence of $h_{c}$ on $R$ was rather close to the change in $h_{c}$ upon a uniform variation of resistivity (dashed line). An entirely different result was obtained when the strand resistivity was altered (Panel B). In this case, the increase of resistivity resulted in an increase of $h_{c}$.

\subsection{Modeling of geometrical transition in heart cell cul-} ture

Recently, $h_{c}$ was measured in 2-dimensional monolayers of cultured neonatal rat heart cells consisting of narrow strands abruptly diverging into a large cell area [19]. In order to test the predictive value of the model, the model was adjusted to fit the morphological and electrophysiological properties of cultured cell strands and monolayers. Two structural features of experimental preparations were simulated. Firstly, the geometrical transition from a strand into the large area was modified to include a funnel, i.e. a final portion of the strand with gradually increasing width. In cell cultures, the funnel had a length of $100 \mu \mathrm{m}$ and a width of about $100 \mu \mathrm{m}$ immediately before the large area. Secondly, a difference in intracellular resistivity between the strand and the large area was included to reproduce the difference in conduction velocity between the strand and the large area $(34 \mathrm{~cm} / \mathrm{s}$ vs. $25 \mathrm{~cm} / \mathrm{s}$ [19]). In addition, the smaller cell size and the flatter shape in cell cultures compared to adult cells were considered by increasing the surface-to-volume ratio, $S_{i}$. These properties were included in the structure which is shown on Fig. 9A. The funnel had a simple linear shape with a length of $100 \mu \mathrm{m}$ and a width of $120 \mu \mathrm{m}$. The Ebihara-Johnson model with $g N a_{\max }=35 \mathrm{mS} / \mathrm{cm}^{2}$ and $S_{V}=0.67 \mu \mathrm{m}^{-1}$ was used. The resistivity was $400 \Omega \mathrm{cm}$ within the strand and gradually increased to $800 \Omega \mathrm{cm}$ upon transition through the funnel into the large area. During steady-state propagation

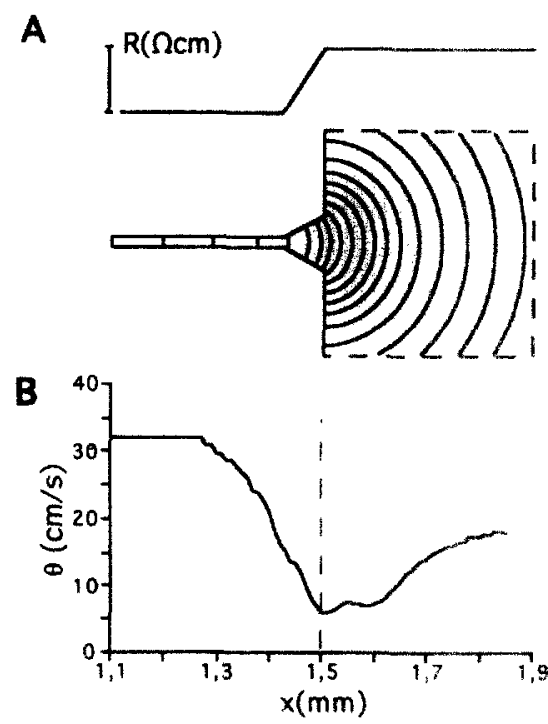

Fig. 9. Impulse conduction in a structure with a terminal funnel and a gradient of $R$. (A) Isochrone map of activation with time interval of 0.3 ms. Strand width $=30 \mu \mathrm{m}$, funnel length $=100 \mu \mathrm{m}$, funnel expansion $=$ from 30 to $120 \mu \mathrm{m}$. Intracellular resistivity increases within the funnel in a linear fashion from $400 \Omega \mathrm{cm}$ to $800 \Omega \mathrm{cm}$. (B) Conduction velocity $(\theta)$ profile across the transition. Ebihara--Johnson model with $\mathrm{gNa} a_{m a . x}=$ $35 \mathrm{mS} / \mathrm{cm}^{2}, S_{r}=0.67 \Omega \mathrm{cm}$. 
the maximal upstroke rate of rise was $172 \mathrm{~V} / \mathrm{s}$, the conduction velocity within the strand and within the large area were $32 \mathrm{~cm} / \mathrm{s}$ and $23 \mathrm{~cm} / \mathrm{s}$, respectively. In these conditions, the critical strand width which resulted in successful propagation was $30 \mu \mathrm{m}$. Panel B demonstrates the profile of conduction velocity upon transition into the large area. In contrast to impulse conduction through a simple step-wise expansion (Fig. 2), conduction velocity was minimal at the transition to the large area which is consistent with experimental findings [19].

\section{Discussion}

\subsection{Evaluation of the computer model}

$1 D$ vs. $2 D$ and $3 D$ simulations. The effect of an abrupt increase in cable diameter on impulse conduction has been extensively studied in $1 \mathrm{D}$ models of axons or of a cardiac strand [5-9]. Two differences exist between a 1D cable and multidimensional (2D and 3D) models. Firstly, in the 1D cable with an abrupt increase in diameter (from $h$ to $h^{\prime}$ ), impulse conduction through the transition region depends on the ratio $h^{\prime} / h$, not on the real values of $h^{\prime}$ or $h$ $[6,7]$. In contrast, in multidimensional models, propagation is dependent on the real value of the strand diameter. Therefore a critical diameter, $h_{\mathrm{c}}$, can be obtained instead of the critical ratio $h^{\prime} / h$. Another difference between conduction in $1 \mathrm{D}$ versus conduction in $2 \mathrm{D} / 3 \mathrm{D}$ models is related to the effect of axial resistivity. In the $1 \mathrm{D}$ case, a uniform change of resistivity $R$ does not affect impulse conduction through the expansion and does not change the critical ratio $h^{\prime} / h$, while an uniform increase of $R$ in 2D and $3 \mathrm{D}$ models facilitates impulse conduction and reduces $h_{c}$ (Fig. 7A). This difference is due to the presence of a transverse resistivity in the $2 \mathrm{D}$ and $3 \mathrm{D}$ models (Fig. 7B).

Comparison of different ionic models. We obtained essentially the same values for $h_{c}$ in all three ionic models of ventricular excitation when excitabilities were adjusted to give comparable values for the maximal upstroke rate of rise. In addition, the values for conduction velocities were close in the EJ and LR models. This is not surprising since these two models share a very similar description for the fast sodium current which is the most important current during the action potential upstroke. There are two differences (besides the differences in $g N a_{\max }$ ) in the sodium current kinetics between LR and EJ models: (1) the $j$ variable in the LR model which describes the kinetics of sodium current reactivation, and (2) the more positive reversal potential, $E_{\mathrm{Na}}$, in LR model as compared to EJ model. The $j$ variable does not change during the upstroke of an action potential and therefore this difference is not important for the depolarisation phase. The higher value of $E_{N a}$ in LR model as compared to EJ model (54 mV vs. 29 $\mathrm{mV}$ ) results in a bigger driving force for the sodium current and, hence, in faster depolarisation. However, this increase in the sodium current is compensated by the smaller $g N a_{\max }$. Other differences between the two models include a slightly more positive resting membrane potential and a smaller membrane resistance in the LR model as compared to the EJ model. The higher resting potential in the LR model accelerates membrane depolarisation. Again, this effect is compensated by adjustment of $g N a_{\text {max. }}$.

The similarity between the EJ and LR models is valid only for propagation of a single wave or for very slow stimulation rates. Different results can be expected at high excitation rates because significant differences exist between EJ and LR models in the formulation of ionic currents during the repolarisation phase.

Continuity of intracellular resistivity. The facilitating effect of increasing intracellular resistivity on impulse conduction across an abrupt expansion and the corresponding decrease of $h_{c}$ (Fig. 7) is limited to tissue with continuous uniform properties. In real myocardium, internal resistivity is distributed nonuniformly between the cytoplasm and the intercellular junctions. When cells are well coupled, the effect of the nonuniformity is likely to be small and the continuous cable equation adequately describes impulse propagation on a macroscopic scale [8,33-35]. However, when intercellular coupling decreases, the nonuniform distribution of resistivity must be taken into account at some level of uncoupling. Thus our model may not be appropriate for certain pathophysiological situations when $R$ increases beyond a critical value. A significant deviation from the behaviour of a continuous cable was observed when axial resistivity increased about five-fold in the one-dimensional computer model [35]. In conditions of an additionally reduced excitability such as in myocardial ischemia, linear cable analysis became invalid when $R$ increased approximately by $200 \%$ [36].

Another limitation of this model is related to the assumption that the resistance of the extracellular space is negligible. Such an assumption is valid in the case of superfused cultured cell monolayers [19], and to some extent in the case of a superfused isolated thin sheet of epicardial tissue. In case of anisotropic tissue with a restricted extracellular space, the results of this model would be quantitatively applicable when the anisotropic ratios for intra- and extracellular resistivities are equal.

\subsection{Estimation of critical strand width in ventricular tissue and cell cultures}

One of the main goals of this study was to estimate $h_{c}$ quantitatively for cardiac tissue. Since the ionic models used in these simulations were developed to describe excitation dynamics of ventricular myocytes, we discuss below two types of ventricular tissue where theoretical and experimental attempts to evaluate critical size were made previously: ventricular myocardium and cultured cell monolayers.

Ventricular myocardium. There is relatively close agreement among the computations of the different types of "critical sizes" in ventricular myocardium. Tyson and Keener estimated the critical diameter of a tissue nucleus which needs to be excited by a central stimulus to get a centrifugal propagated response. Using a theory which assumes a linear dependence of the velocity of activation 
front on front curvature they obtained a value of $200 \mu \mathrm{m}$ [23]. Winfree estimated the same parameter from strengthduration curves and arrived at a value of $300 \mu \mathrm{m}$ for $3 \mathrm{D}$ tissue [24]. The other critical parameter, the "liminal length"' (size of a tissue area generating inward membrane current during point stimulation $[20,21]$ ) was calculated by Joyner et al. in the 2-dimensional Beeler-Reuter model and values between $200 \mu \mathrm{m}$ and $370 \mu \mathrm{m}$ were found for different levels of $\mathrm{gNa}_{\max }$ [22]. We have previously reported a value of $300 \mu \mathrm{m}$ for the critical strand width in the isotropic 2D Ebihara--Johnson model [37]. The slightly smaller critical strand width obtained in the present study in different ionic models $(175-200 \mu \mathrm{m})$ is due to the larger assumed value for the surface-to-volume ratio and, hence, a smaller space constant. Cabo et al. calculated the critical width of a short isthmus connecting two large excitable areas [16]. Comparison of the "isthmus geometry" with the "strand geometry" yielded a $25 \%$ smaller value of $h_{c}$ in the strand (see Figs. 2 and 5). The difference between $h_{c}$ in the two geometries is explained by the acceleration of conduction and the increase in action potential amplitude in front of the isthmus. In the case of the diverging strand, no increase in conduction velocity or amplitude was present (Fig. 2B). To calculate $h_{c}$ in anisotropic tissue, the orientation of the longitudinal cell axis with respect to the direction of propagation must be taken into account. Depending on cell orientation, anisotropy will reduce $h_{c}$ by a factor of 1 to 3 if a ratio of 10 is assumed for anisotropic differences between $R_{x}$ and $R_{y}$ [38]. This factor is derived from extrapolation of the dependence $h_{c}\left(R_{y}\right)$ shown on Fig. 7 to the $R_{y}$ value of $5000 \Omega \mathrm{cm}$. As a whole, the estimates for the different values of critical size vary between 60 and $200 \mu \mathrm{m}$ in 2-dimensional tissue.

For the 3-dimensional tissue, our results predict a value 2.7 times larger than in 2-dimensional case (Fig. 6A and 7A). Thus, $h_{c}$ in anisotropic 3D tissue is in the range of $160-500 \mu \mathrm{m}$. This is consistent with the estimations of critical size of excitation as mentioned above. All estimates are significantly larger than the diameter of terminal Purkinje fibers which can be less than $100 \mu \mathrm{m}$ [39]. Several factors may be taken into account to explain this discrepancy. Firstly, the intracellular longitudinal resistivity of Purkinje fibers has been reported to be lower than in the working myocardium [34,40,41]. According to our simulations (Fig. 8A) this difference will reduce $h_{c}$. Secondly, the funnel hypothesis [11] postulated the presence of a gradually expanding portion within the PM junction which facilitates impulse transmission. Thirdly, conduction may be facilitated by the presence of a resistive barrier between the two tissues which may reduce the load of large ventricular mass on Purkinje fibers [7,42].

Cell culture. Recently, $h_{c}$ was measured in cultured cell monolayers [19]. In these experiments, propagation was investigated in long cell strands of variable width diverging into a large cell area, i.e. in tissue with a geometry analogous to the simulated structures. In such cultures, $h_{c}$ was $30 \mu \mathrm{m}$ which is considerably lower than the computed 2-dimensional $h_{c}$ value for a simple step-wise expansion. However, when the specific geometrical and func- tional properties of cultured cell monolayers were taken into account, a close quantitative agreement was obtained.

\subsection{Effect of tissue inhomogeneities}

In general, success or failure of impulse conduction depend on the electrical properties of both the strand (current source) and the large area (current sink). Several situations correspond to a case where the passive and active properties in the strand differ from the large area. One example is the junction between a Purkinje fiber and ventricular muscle. Another example is represented by cultured cell monolayers where cell strands and the large area have different intracellular resistivities between two parts $[18,19]$. Our results demonstrate that there is a considerable difference between the contributions of the source and the sink in impulse transmission at a geometrical expansion: varying maximum sodium conductance in the strand had little effect on impulse propagation in comparison to the changes in excitability of the large area (Fig. 6). We can conclude that the critical size is mainly determined by the excitable properties of the large area. This simplifies the modelling of the expansion region since only the excitable properties of the large region need to be simulated precisely. The strand $\mathrm{gNa}_{\max }$ does not substantially influence $h_{c}$ because the amount of axial current supplied by the strand into the large area is mainly dependent on the voltage difference between the two parts which is equal to the action potential amplitude. The action potential amplitude does not change significantly with variation of $g N a_{\text {max }}$.

In contrast to the changes in excitability, the effects of changes in resistivity in the strand and in the large area were opposite (Fig. 8). The increase in the resistivity of the large area facilitated impulse conduction across the expansion and reduced critical strand width. This is explained by the reduction of the electrical load imposed by the large area on the strand. In contrast, the increase of the strand resistivity impaired impulse conduction across the expansion. The explanation for the latter effect is that the large strand resistivity reduced the amount of axial current flowing from the strand into the large area. A difference in resistivity between the strand and the large area may contribute to the success of impulse conduction at the PM junction as discussed above.

\subsection{Critical curcature or impedance mismatch?}

It has been suggested that conduction slowing and block at the site of a geometrical expansion are determined by the curvature of the excitation front emerging from the narrow isthmus rather than by the impedance mismatch between the small and the large parts [16]. Following this line of reasoning, it can be suggested that $h$ is determined by a critical value for the curvature in the large area. Our results partly support this point of view. In particular, the block of conduction from a strand of subcritical width occurred beyond the transition (Fig. 3). Since a small area of cxcitation was formed in the large area, the dissipation of excitatory current from a highly curved excitation front contributed to the conduction block. Also, the finding that 
the changes in $h_{c}$ were largely independent of the strand excitability and were mainly attributed to the modification of $g N a_{m a x}$ in the large area is in agreement with this idea. Several considerations indicate that the curvature alone does not fully describe all changes related to the geometrical expansion, however. Firstly, the conduction block in a very narrow strand may occur without the excitation wave entering the large area (Fig. 4). Since the curvature of the excitation front did not change within the strand, the block in this case can be explained by the impedance mismatch between the strand and the large area alone. Secondly, the consideration of the critical curvature does not correctly predict differences of critical strand widths in 3-vs. 2-dimensional models. Indeed, the value of curvature of a spherical wave front in a 3-dimensional medium is two times larger than the curvature of a circular front in a 2 -dimensional medium with the same radius. Therefore, critical strand width should be two times larger in the $3 \mathrm{D}$ than in the 2D model. However, in our simulations the 3D-to-2D ratio of $h_{c}$ was 2.7. The stronger dependence of the critical strand width on tissue dimensionality than predicted from the curvature hypothesis suggest that the impedance mismatch between the strand and the large area contributes substantially to conduction changes at the geometrical expansion. Thus, both the front curvature and the impedance mismatch appear to determine the geometry-dependent slowing of conduction and conduction block at the site of abrupt geometrical expansion.

\section{Acknowledgments}

This work was supported by the Bonizzi-Theler Foundation the Swiss Heart Foundation, and the Swiss National Science Foundation

\section{References}

[1] Mendez, C, Mueller, WJ, Merideth J, Moe GK. Interaction of transmembrane potentials in canine Purkinje fibers and at Purkinje fiber-muscle junctions. Circ Res 1969;34:361-372.

[2] Joyner RW, van Capelle FJL. Propagation through electrically coupled cells. How a small SA node drives a large atrium. Biophys J 1986;50:1157-1164.

[3] De la Fuente D, Sasyniuk B, Moe GK. Conduction through a narrow isthmus in isolated canine atrial tissue. A model of the W-P-W syndrome. Circulation 1971;54:803-809.

[4] Smith JH, Green CR, Peters NS, Rothery S, Severs NJ. Altered patterns of gap junction distribution in ischemic heart disease - an immunohistochemical study of human myocardium using laser scanning confocal microscopy. Am J Pathol 1991;139:801-821.

[5] Khodorov BI, Timin YN, Vilenkin SY, Gul'ko FN. Theoretical analysis of the mechanisms of conduction of a nerve pulse over an inhomogeneous axon. I. Conduction through a portion with increased diameter. Biofizika 1969;14:304-315.

[6] Goldstein SS, Rall W. Changes of action potential shape and velocity for changing core conductor geometry. Biophys J 1974;14:731-757.

[7] Joyner RW, Veenstra R, Rawling D, Chorro A. Propagation through electrically coupled cells. Effects of a resistive barrier. Biophys $\mathrm{J}$ 1984;45:1017-1025.

[8] Henriquez CS, Plonsey R. Effects of resistive discontinuities on waveshape and velocity in a single cardiac fibre. Med Biol Eng Comput 1987;25:428-438.
[9] Quan W, Rudy Y. Unidirectional block and reentry of cardiac excitation: a model study. Circ Res 1990;66:367-382.

[10] Sahakian AV, Myers GA, Maglaveras N. Unidirectional block in cardiac fibers - effects of discontinuities in coupling resistance and spatial changes in resting membrane potential in a computer simulation study. IEEE Trans Biomed Eng 1992;39:510-522.

[11] Mendez C, Muller WJ, Urguiaga X. Propagation of impulses across the Purkinje fiber - muscle junctions in the dog heart. Circ Res 1970;26:135-150.

[12] Sasyniuk BI, Mendex C. A mechanism for recntry in caninc ventricular tissue. Circ Res 1971;28:3-15.

[13] Overholt ED, Joyner RW, Veenstra RD, Rawling D, Wiedmann R. Unidirectional block between Purkinje and ventricular layers of papillary muscle. Am J Physiol 1984;247:H584-H595.

[14] Spach MS, Miller WTI, Dolber PC, Kootsey JM, Sommer JR, Mosher CE. The functional role of structural complexities in the propagation of depolarization in the atrium of the $\mathrm{dog}$. Cardiac conduction disturbances due to discontinuities of effective axial resistivity. Circ Res 1982;50:175-191.

[15] Inoue H, Zipes DP. Conducton over an isthmus of atrial myocardium in vivo: a possible model of Wolf-Parkinson-White syndrome. Circulation 1987;76:637-647.

[16] Cabo C, Pertsov AM, Baxter WT, Davidnko JM, Gray RA, Jalife J. Wave-front curvature as a cause of slow conduction and block in isolated cardiac muscle. Circ Res 1994;75:1014-1028.

[17] Fast V, Kléber A. Tissue geometry as a determinant of unidirectional conduction block: assessment of microscopic excitation spread by optical mapping in patterned myocyte cultures. Circulation 1993;88:I-623.

[18] Rohr S, Salzberg BM. Charactertization of impulse propagation at the microscopic level across geometrically defined expansions of excitbale tissue: multiple site optical recording of transmembrane voltage (MSORTV) in patterned growth heart cell cultures. J Gen Physiol 1994;104:287-309.

[19] Fast V, Kléber A. Cardiac tissue geometry as a determinant of unidirectional conduction block: assessment of microscopic excitation spread by optical mapping in patterned cell cultures and in a computer model. Cardiovasc Res 1995;29:697-707.

[20] Noble D. The relaton of Rushton "liminal length" for excitation to the resting and active conductances of excitable cells. J Physiol 1972;226:573-591.

[21] Fozzard HA, Schoenberg M. Strength-duration curves in cardiac Purkinje fibres: effects of liminal length and charge distribution. J Physiol 1972;226:593-618.

[22] Ramza BM, Joyner RW, Tan RC, Osaka T. Cellular mechanism of the functional refractory period in ventricular muscle. Circ Res 1990;66:147-162.

[23] Tyson JJ, Keener JP. Singular perturbation theory of traveling waves in excitable media. Physica D 1988;32:327-361

[24] Winfree AT. The electrical thresholds of ventricular myocardium. J Cardiovasc Electrophysiol 1990;1:393-410.

[25] Beeler GW, Reuter H. Reconstruction of the action potential of ventricular myocardial fibres. J Physiol 1977;268:177-210.

[26] Ebihara L, Johnson EA. Fast sodium current in cardiac muscle. A quantitative description. Biophys J 1980;32:779-790.

[27] Luo CH, Rudy Y. A model of the ventricular cardiac action potential - depolarization, repolarization, and their interaction. Circ Res 1991;68:1501-1526.

[28] Peaceman DW, Rachford HH. The numerical solution of parabolic and elliptic differential equations. J Soc Indust Appl Math 1955;3:28-41.

[29] Rush S, Larsen H. A practical algorithm for solving dynamic membrane equations. IEEE Trans Biomed Eng 1978;BME-25:389392.

[30] Victorri B, Vinet A, Roberge FA, Drouhard J-P. Numerical integration in the reconstruction of cardiac action potentials using Hodgkin-Huxley-type models. Computers in Biomed Res 1985;18:10-23.

[31] Alanis J, Benitez D. Transitional potentials and the propagation of impulses through different cardiac cells. In: Sano, T, Mizuhira, V, Matsuda, $K$ eds. Electrophysiology and Ultrastructure of the Heart. Tokyo: Bunkodo, 1967:153-175. 
[32] Bukauskas FF, Sakson MY, Kukushkin NI. Discrete zones of the electrical connexion of Purkinje terminals with muscle fibres in the canine ventricle. Biofizika 1976;21:887-892.

[33] Joyner RW. Effects of the discrete pattern of electrical coupling on propagation through an electrical syncytium. Circ Res 1982;50:192 200.

[34] Kléber AG, Riegger CB. Electrical constants of arterially perfused rabbit papillary muscle. J Physiol (Lond) 1987;385:307-324.

[35] Rudy Y, Quan W. A model study of the effects of the discrete cellular structure on electrical propagation in cardiac tissue. Circ Res 1987;61:815-823.

[36] Kléber $A G$, Riegger $C B$, Janse $M J$. Electrical uncoupling and increase of extracellular resistance after induction of ischemia in isolated, arterially perfused rabbit papillary muscle. Circ Res 1987;61:271-279.

[37] Fast VG, Kléber AG. Current-to-load mismatch in Purkinje-muscle junction: evaluation of critical fiber diameter by 2-dimensional simulation. Circulation 1991;84(suppl II):II-325 (abstract).

[38] Clerc L. Directional differences of impulse spread in trabecular muscle from mammalian heart. J Physiol (Lond) 1976;255:335-346.

[39] Glomset DJ, Glomset ATA. A morphology study of the cardiac conduction system in ungulates, dog, and man. Am Heart J 1940;20:677-701.

[40] Weidmann S. The electrical constants of Purkine fibres I Physiol (Lond) 1952;118:348-360.

[41] Weidmann S. Electrical constants of trabecular muscle frum mammalian heart. J Physiol (Lond) 1970;210:1041-1054.

[42] Tranum-Jensen J, Wilde AAM, Vermeulen JT, Ianse MJ. Morphology of electrophysiologically identified junctions between Purkinje fibers and ventricular muscle in rabbit and pig hearts. Circ Res 1991:69:429-437 